\title{
A SECURE WORKFLOW SYSTEM FOR DYNAMIC COLLABORATION
}

\author{
Joon S. Park, Myong H. Kang, and Judith N. Froscher \\ Center for High Assurance Computer Systems \\ US Naval Research Laboratory \\ \{jpark, mkang, froscher\} @ Itd.nrl.navy.mil
}

\begin{abstract}
The emergence of the Internet has broken down geographic and organizational boundaries, providing a virtual common workplace regardless of the heterogeneity of participating organizations. Enterprise projects that used to be done autonomously now span multiple organizations. While an inter-organizational workflow, as one of several technologies supporting inter-organizational collaboration, provides an easy-to-use collaborative work environment for users, it also increases the complexity of security maintenance and brings about security problems that were not considered before. Unconventional collaborations among businesses and organizations are formed to advance common goals. In this paper, we address the security services to support inter-organizational collaborative enterprises, which may span multiple organizations, and describe how we develop a secure workflow system to satisfy the requirements by integrating with existing, wellknown technologies. Although we apply our ideas to particular technologies, such as workflows and RBAC, in this paper, we believe it is always possible to apply our approaches to other systems, which support many users from different organizations.
\end{abstract}

Keywords: Dynamic Collaboration, Information Security, Role-based Access Control (RBAC), Workflow

\section{INTRODUCTION}

In the days before the ubiquitous Internet and its use across all industries, collaborative projects were planned in accordance with geographic and organizational borders. The emergence of the Internet has broken down these boundaries, providing a virtual common workplace. Organizations can communicate with suppliers and partners, and with customers more efficiently and effectively. Enterprises that were autonomous now span multiple organizations, which may 
join or leave an enterprise project dynamically while the project is still underway.

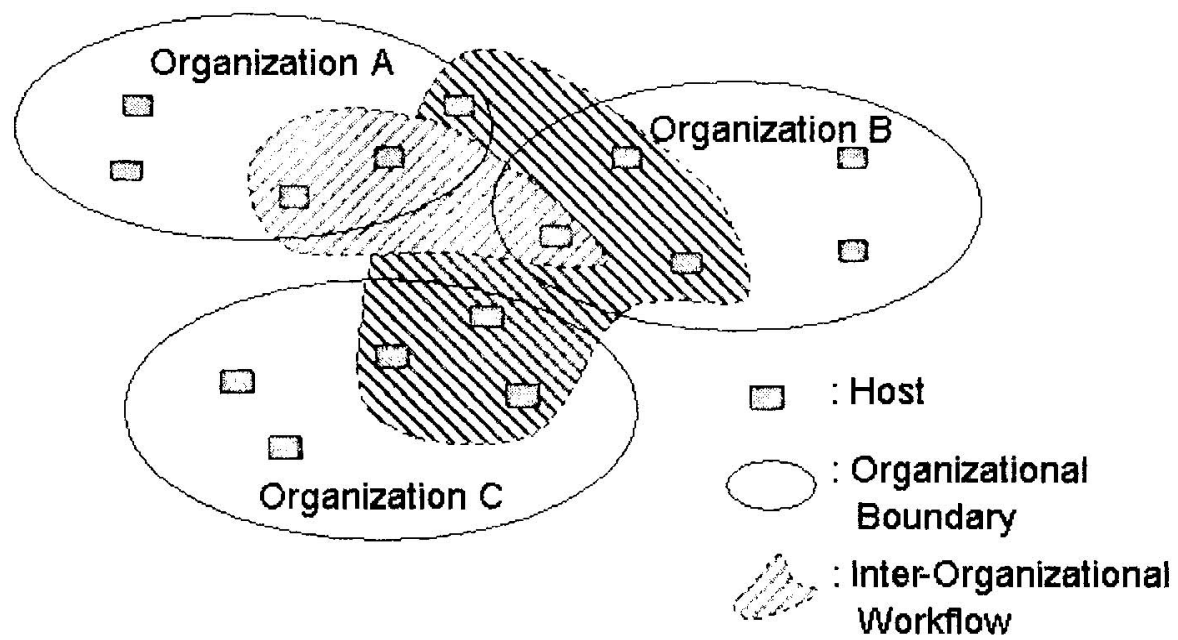

Figure 1 An Example of Inter-Organizational Workflows

One technology that can satisfy this service is inter-organizational workflow. We consider an inter-organizational workflow as a virtual enterprise in this paper. Figure 1 shows an example of inter-organizational workflows, which span multiple organizations to conduct their missions. In this example, one workflow spans two organizations while the other workflow spans three organizations. Once a workflow is designed, each task is conducted in a specific host (machine) in a specific organization. The hosts are connected via the Internet and may support multiple tasks for multiple enterprises (particularly, workflows in this paper). Individual users conduct their human tasks by connecting to a specific machine in a specific domain, while non-human tasks are executed automatically on demand under the workflow policy.

While an inter-organizational workflow supports an easy-to-use collaborative work environment for users, it also increases the complexity of security maintenance and causes new security problems that did not appear in autonomous enterprises. For example, how can we control efficiently and securely who is doing what and when? Unconventional collaborations among businesses and organizations are formed to advance common goals. These collaborations may quickly dissolve as individual objectives change (we call these dynamic collaborations in this paper). Threats now lie in these essential connections among participating organizations, which may be involved in multiple enter- 
prises across other organizations. Therefore, there is the need for new types of security services for the common workplace, which provides a collaborative work environment.

For a secure workflow, especially, if dynamic collaboration is necessary, we need the following security services.

- Providing secure communication between components and users

- Separating security infrastructures between organizations and enterprises (workflows)

- Providing different privileges to different users

- Validating enterprise (workflow) design

In this paper, we describe why we need to satisfy the above requirements for a dynamic workflow system, and how we have implemented each in our system by integrating with existing security technologies. Although we describe our approaches within a workflow management system that we have developed, we believe that the technologies we introduce in this paper can be easily applied to other systems, which support many users, requiring security services between components and users.

The rest of this paper is organized as follows. In Section 2, we briefly introduce the existing technologies that we use for our implementation, including OrbWork, RBAC (Role-based Access Control), and SSL (Secure Socket Layer). Section 3 describes the system architecture of our secure workflow management system. In Section 4, we describe how we provide security services to our workflow system for dynamic collaboration. Finally, Section 5 summarizes our implementation and concludes this paper.

\section{RELATED WORK}

\subsection{ORBWORK}

Researchers at the University of Georgia developed a workflow enactment service, OrbWork [10] in 1998. OrbWork is a single-level distributed workflow engine that exploits CORBA (Common Object Request Broker Architecture), JAVA, and Web technologies. It does not have a central scheduler; rather it is distributed with a scheduler per task. Each scheduler only knows its predecessors and successors.

Basically, OrbWork consists of the following CORBA servers: task servers, worklist servers, and data servers. Figure 2 shows how the OrbWork components interact with each other. Each task server may contain more than one task. Each task has three parts: task scheduler, task manager and the underlying component. The worklist server maintains the lists of pending work for 


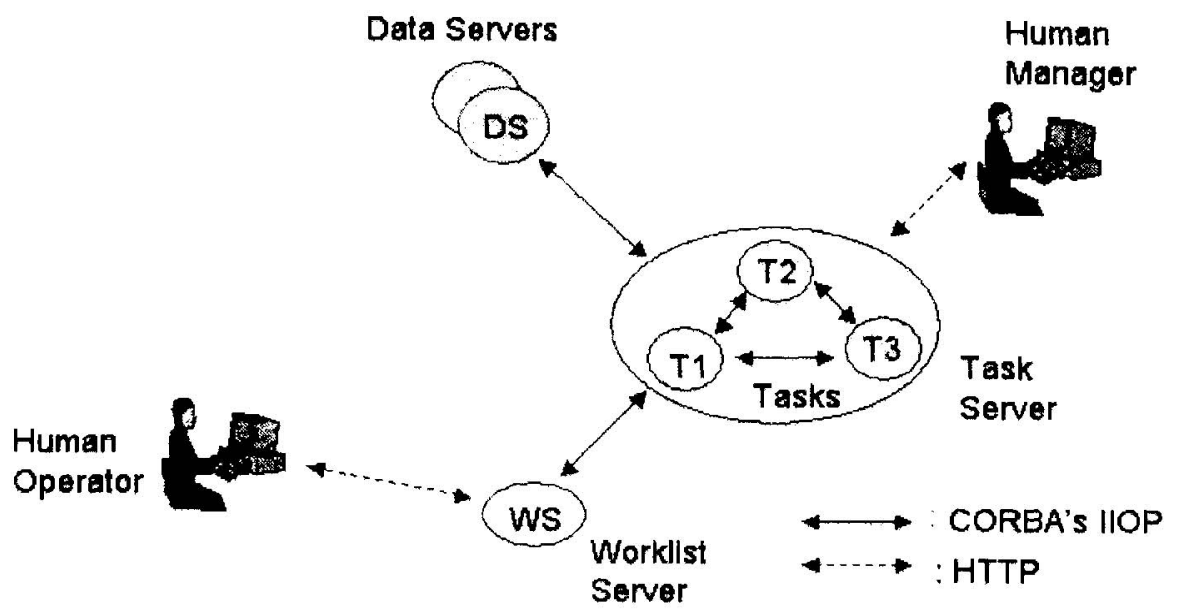

Figure 2 Communications Among the OrbWork Components

human tasks. Data servers act as repositories for data that need to be accessed by tasks. Since they are CORBA servers, they communicate with each other through CORBA's IIOP (Internet Inter-ORB Protocol).

The task and worklist servers are not only CORBA servers but also HTTP (HyperText Transfer Protocol) servers. When a human operator has to interact with the worklist server (e.g., human task), he can do so through HTTP. Also when a human workflow manager needs to intervene in task servers for some reason, he can do so through HTTP. Currently, the original OrbWork does not provide security services among its components and between its components and users.

\subsection{ROLE-BASED ACCESS CONTROL}

A large workflow system is usually designed to support many users. Some users need to be temporarily involved in the workflow. A user may need to have different access privileges based on his context, while other users may need to have the same privilege. If we use the conventional identity-based access control mechanism, it is very hard to determine and control which permissions should be authorized for what users, especially, in a large system. The direct mapping between users and permissions is transitory and brings very inefficient management. Therefore, we have decided to use Role-based Access Control (RBAC [18]) for our secure workflow management system for dynamic collaboration. 


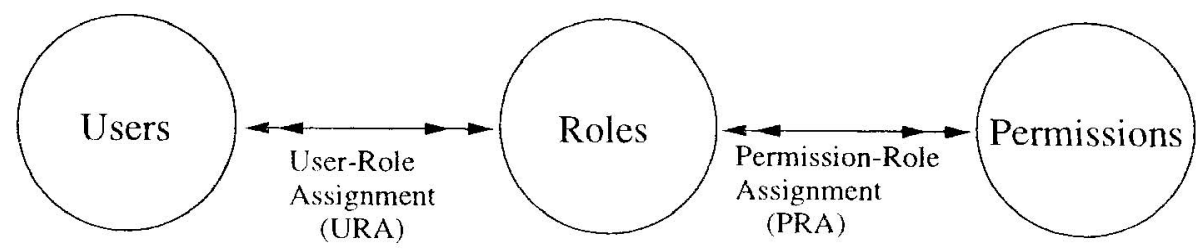

Figure 3 A Simplified RBAC Model

RBAC has rapidly emerged in the 1990s as a technology for managing and enforcing security in large-scale enterprise-wide systems. The basic notion of RBAC is that permissions are associated with roles, and users are assigned to appropriate roles thereby acquiring the roles' permissions. Figure 3 shows a simplified RBAC model. RBAC ensures that only authorized users are given access to certain data or resources.

In RBAC, a role is a semantic construct forming the basis of an access control policy. System administrators can create roles, grant permissions to those roles, and then assign users to the roles on the basis of their specific job responsibilities and policy.

RBAC separates the mapping between users and permissions through UserRole Assignment (URA) and Permission-Role Assignment (PRA). Usually, PRA is more stable (of course it can be changed if it is necessary) than URA, because job responsibilities in an organization do not change frequently while users' job functions change quite often. The system makes access control decisions based on the users roles instead of their identities. This provides an efficient access control mechanism to the system and resolves the scalability problem.

To implement the RBAC model on the Web, Park and Sandhu have identified two different approaches for obtaining a user's roles, especially, with respect to user-pull and server-pull architectures [13]. Basically, there are three components in both architectures: client, Web server, and role server. Clients connect to Web servers via HTTP using browsers. The role server is maintained by an administrator and assigns users to the roles in the domain. In the user-pull architecture, a user pulls his roles from the role server and then presents them to the Web servers. In the server-pull architecture, each Web server pulls the user's roles from the role server as needed and uses them for RBAC. Comprehensive descriptions and tradeoffs between the two different RBAC architectures are discussed in [16]. In this paper, we apply those approaches to build our secure workflow system, providing RBAC services in individual task servers (described in Section 2.1). Detailed technologies (such as authentication, role 
transfer and protection, and verification) to support these architectures depend on the applications and environments.

\subsection{SECURE SOCKET LAYER (SSL) PROTOCOL}

The SSL protocol [21] was introduced with the Netscape Navigator browser in 1994, and rapidly became the predominant security protocol on the Web. Since the protocol operates at the transport layer, any program that uses TCP (Transmission Control Protocol) is ready to use SSL connections. The SSL protocol provides a secure means for establishing encrypted communication between Web servers and browsers. SSL also supports the authentication service between servers and clients.

SSL uses X.509 [4] certificates. Server certificates provide a way for clients to authenticate the identity of a server. The client uses the server's public key to negotiate a secure TCP connection with the server. Optionally, the server can authenticate clients by verifying the contents of the clients' certificates.

Even though SSL provides secure communications between servers and clients, it cannot protect against end-system threats [14]. For instance, if a user receives sensitive information from the server over a secure channel, it does not mean that the information is saved securely in the user's machine. In other words, once the user receives the information from the server over the secure channel, he is able to change the information or give it to other people, because SSL does not support security services in the user's end system. However, as we will see later in this paper, SSL can be used as part of our solution to protect information in our implementation.

\section{SYSTEM ARCHITECTURE OF SECURE WORKFLOW MANAGEMENT SYSTEM}

In this section, we describe the system architecture of our secure workflow management system for dynamic collaboration based on our implementation. There are five major components in the system: design tool, policy server, runtime engine, monitor, and users. Figure 4 shows the components and their relationships in the system. Detailed descriptions about the implementation of this architecture are available in $[7,8]$.

The design tool allows workflow designers to design independent workflows and express their global and local policies and constraints. Global policies and constraints (e.g., User-Role Assignment (URA)) are transferred to the policy server and applied to the whole system. "Global" can be translated from a whole workflow (enterprise) to the whole system, which supports multiple workflows (enterprises). Local policies and constraints (e.g., Permission-Role Assignment (PRA)) are transferred and applied to only relevant tasks in the runtime engine autonomously. Technically, it is always possible to enforce URA locally or 


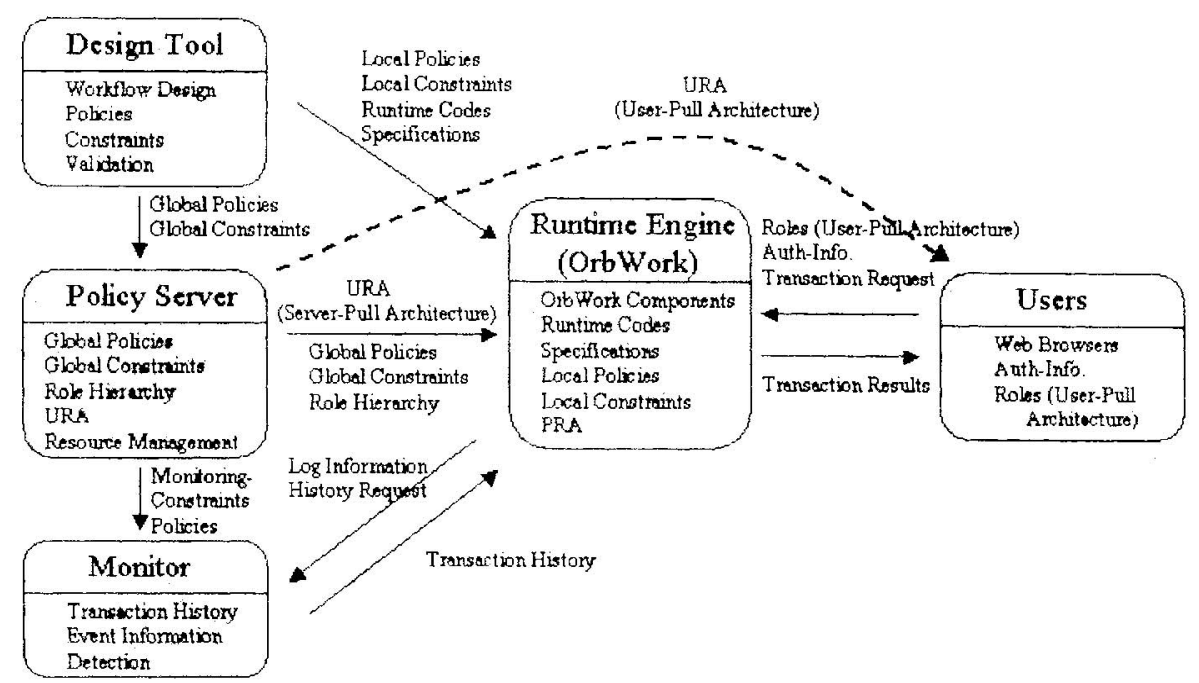

Figure 4 System Architecture of Secure Workflow Management System

PRA globally. Furthermore, different workflows (enterprises) may have different URAs and PRAs. However, we believe that our policy enforcement (global URA and local PRA) is efficient for maintaining organizational consistency and providing autonomy of tasks in the runtime engine. The design tool also validates if a workflow design is consistent and sound. After the designer finishes workflow design, the design tool generates runtime codes and specifications for the workflow that will be used in the runtime engine.

The policy server provides global policies and constraints to the other components in the system. For instance, it enforces URA or resource management for the whole system. A typical component in the policy server is a role server. The role server provides role hierarchy and URA information to support RBAC in the system. In the server-pull RBAC architecture (see Section 2.2), individual task servers connect to the role server and pull the user's roles on demand. In the user-pull RBAC architecture (see Section 2.2), the user connects to this role server and pulls his roles after proper authentication procedures (denoted by a dotted line in Figure 4). Later, he uses those roles in the task servers in the runtime engine to execute human tasks. Technically, a single user-credential can be issued by the policy server and used for both authentication and authorization in the runtime engine. For instance, the policy server can issue an X.509 certificate for the user including the user's roles and public-key information. Once the user pulls this certificate, he can use it to prove his identity and roles in 
the task servers. However, we do not claim that this kind of bundled certificate is always good. Especially, if the lifetimes of a user's role and public-key information are different, or if different authorities must issue the role and identity information, bundled certificate may not be a good solution. Instead, we can use two different certificates to satisfy the above requirements. In this case, we must support the binding of attributes (e.g., roles) and identification for each user [15]. For instance, if Alice presents Bob's roles with her authentication information to the Web server, she must be rejected. It is important to note that the policy server does not have local policies and constraints, which are defined by the design tool and enforced by the individual tasks in the runtime engine.

The runtime engine consists of OrbWork (described in Section 2.1) components (task servers, worklist servers, and data servers), PRA, and the information generated by the design tool, such as runtime codes, specifications, local policies, and local constrains. It conducts the workflow tasks, including human tasks and non-human tasks, using the OrbWork components in conjunction with the runtime codes and specifications generated by the design tool. During installation and execution, the runtime engine refers to the PRA, local policies and constraints that it has, and the URA, global policies and constraints that the policy server provides. The runtime engine also refers to the monitor to get the transaction history and make a correct decision. It is important to note that the runtime engine does not have global policies and constraints, which are defined by the design tool and enforced by the policy server.

The monitor consists of a monitor server and client. The monitor server receives event information from the runtime engine and records it in a file. The monitor server has application-layer monitoring functions that provide event information, based on its clients' interests. Furthermore, the monitor server provides the transaction history to the runtime engine (if it is necessary) so that the runtime engine can make a correct decision that complies with the policies and constraints based on the user's previous transaction history [2]. Inter-organizational workflows may consist of several autonomous workflows. Hence, there may be multiple monitor servers. In our implementation, there is a monitor server per runtime engine. Each monitor server refers to the policy server for its monitoring policy and constraints, and has its own database so that it can record events from OrbWork and answer any query from OrbWork or monitor clients. Monitor clients can register their topics of interests to monitor servers. For example, one monitor client may be interested in all events in a specific workflow while another monitor client may be interested in only events that have to do with a specific task. The monitor server records clients' interests and dispatches only those events that each client is interested in.

In our system, users communicate with the runtime engine using Web browsers via HTTP or HTTPS. Users are assigned to their roles in the policy server (particularly, role server) under the enterprise policy. When a user connects to 
the runtime engine using a Web browser, the runtime engine authenticates the user by means of existing authentication mechanisms such as passwords, Kerberos [19], X.509, and so on. In the user-pull RBAC architecture, the user's role information is transferred to the runtime engine from the user's machine (assuming that the user pulled his roles from the role server before). In the server-pull RBAC architecture, the runtime engine pulls the user's role information from the role server after it authenticates the user.

\section{SECURITY SERVICES FOR DYNAMIC COLLABORATION}

In Section 3, we describe the architecture of our secure workflow management system. Each component in the system may be involved in multiple workflows, which may span multiple organizations. This implies that the complexity of security services for inter-organizational enterprises becomes higher than fully in-house projects. In other words, if more organizations are participating in the enterprise, then more efficient and strong security services are required (sometimes even new security services are required). In this section, we focus on the security services for a secure workflow management system for dynamic collaboration and describe how we have provided those services to our secure workflow systems.

\subsection{SECURE COMMUNICATION}

Basically, there are two different kinds of communications that we need to protect in our system. Firstly, we need to protect the communications between users and the OrbWork components. Secondly, we need to protect the communications among the OrbWork components. There may be many possible technologies and implementations to satisfy those requirements. Since one of our strategies in this work is the maximum use of available COTS security solutions with the minimum modification of the system components, we have decided to use a standard technology, SSL (described in Section 2.3), for our purposes.

The runtime engine (OrbWork) supports both HTTP and IIOP. The former supports the communications between the OrbWork components and users via their Web browsers. The latter supports the communications among the OrbWork components in CORBA, where all objects access other objects or services via Object Request Brokers (ORBs). By integrating an SSL (we used Phaos' SSLava [20] in our implementation) package with OrbWork, we provide HTTPS for the secure communications between users and the OrbWork components, and SSL-IIOP for the secure communications among the OrbWork components in CORBA. We do not describe other alternative security technologies, such as IPSEC [6], SECIOP (Secure Inter-ORB Protocol) using SPKM (Simple Public- 
Key Mechanism [1]), Kerberos [19], or SESAME (Secure European System for Applications in a Multivendor Environment [ 12]), or DCE-CIOP (Distributed Computing Environment - Common Inter-ORB Protocol) using DCE [17], in detail in this paper, since we believe HTTPS and SSL-IIOP are simple and adequate solutions for our purposes.

\subsection{SEPARATING SECURITY INFRASTRUCTURES FOR ORGANIZATIONS AND ENTERPRISES}

When several organizations are involved in a large inter-organizational enterprise, especially, when dynamic collaboration is required, there are several security issues that would not be considered in a static in-house project. First, each organization has its own security infrastructure (e.g., organizational role hierarchy), which is different from others including that of the inter-organizational enterprise. If there is a direct assignment between an organizational role and the permission for the inter-organizational enterprise, changes in an organization role hierarchy requires unexpected changes in PRA (Permission-Role Assignment) for the enterprise. Second, the participating organizations may change during the life cycle of the enterprise. For example, a new organization may replace an old organization or there may be a merger or separation among organizations. In this case, how can we assign or revoke users to or from their job responsibilities (e.g., roles) for the enterprise efficiently? To resolve the above problems, we could change the organization security infrastructure (e.g., role hierarchy in the above example) to fit the enterprise's security infrastructure whenever it is necessary. However, it is not sound for dynamic collaboration to restructure each organization's security infrastructure for a particular inter-organizational enterprise. Usually, the lifetime of an enterprise is shorter than those of participating organizations. Furthermore, each organization may support several different enterprises with others. Therefore, we should insulate the security infrastructures for participating organizations and their inter-organizational enterprises.

To achieve this goal, we introduce a concept of role domain, which is a role structure interface for an inter-organizational enterprise. Figure 5 shows two different cases for managing security structures (role structures in this example) for organizations and their inter-organizational enterprises. The relationship between a role domain and the role structures of organizations is similar to an interface in client-server interactions in a distributed environment. It is each organization's responsibility to map its own role structure to the enterprise's role domain. In this case, the role structures of participating organizations can be managed independently and autonomously form those of the enterprises as depicted in Figure 5(b). One organization may map its own role structure to multiple role domains in different ways if it is involved in different 


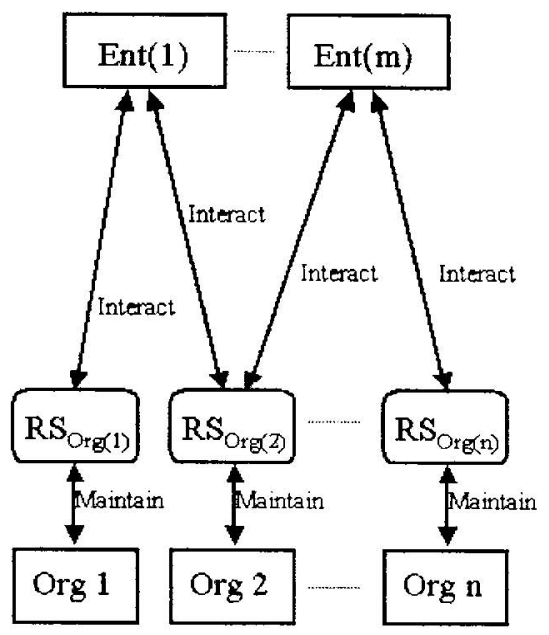

(a) Without Role Domains

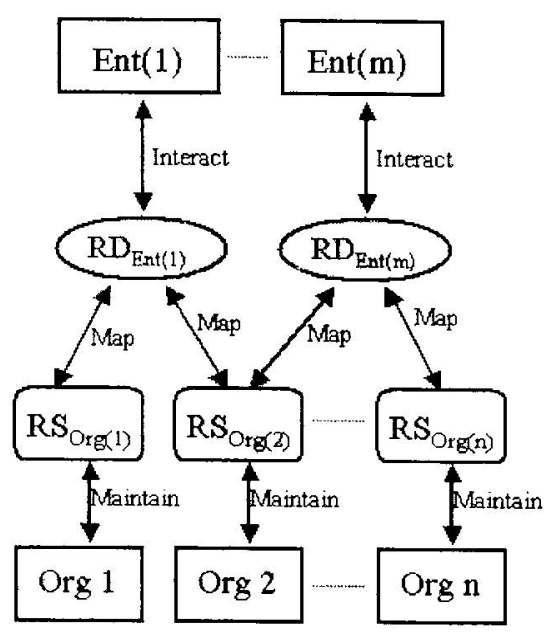

(b) With Role Domains

Org (n): Organization n

$\mathrm{RS}_{\mathrm{Org}(\mathrm{n})}$ : Role Structure for Organization $\mathbf{n}$

Ent(m): Enterprise $m$

$R D_{\text {Ent }(m)}:$ Role Domain for Enterprise m

Figure 5 Managing Security Structures for Organizations and Enterprises

inter-organizational enterprises. The tasks in the individual enterprises require specific roles in its enterprise's role domain for their access control decisions instead of the users' roles in their organizations. Detailed descriptions for the access control mechanisms, including fine-grained and context-based access control with dynamic constraints, within the tasks are available in [9].

\subsection{PROVIDING DIFFERENT PRIVILEGES TO DIFFERENT USERS}

Usually, a large collaborative enterprise spans several organizations, which support a variety of tasks executed by many different users, and consist of many different components, which may change dynamically. It is obvious that individual users - who may belong to different organizations - should have different privileges (roles in our case) for more secure and efficient enterprise management. Therefore, we need to provide different privileges to different users based on the users' needs-to-do in the enterprise. For example, users working on task $\mathrm{T} 1$ need to access the components (tasks) related to $\mathrm{T} 1$, but 
may not need to access (even know the existence of) the components that are irrelevant to $\mathrm{T} 1$ or under the control of other enterprises.

Technically, we could control each user's privileges by the conventional identity-based access control mechanism. This could work for a small project, where a small number of users are involved. However, for a large enterprise, where many users from different organizations join and leave dynamically, the identity-based access control mechanism is inefficient and becomes too complicated to manage. Fortunately, individual users have common job functionalities (abstracted as roles in this paper) for the enterprise. Therefore, we devise a strong and efficient mechanism to provide different privileges to different users by integrating the RBAC model (described in Section 2.2) with our role domain concept (described in Section 4.2), and enforce this mechanism in our system.

In our design tool (see Section 3), we provide a way to specify a required role set (including role domain and roles) for each task in the following format, where $R D_{m}$ is a specific role domain and $R_{m n}$ is a specific role in the role domain $R D_{m}$.

$$
\begin{aligned}
& {\left[\left\{R D_{1}:\left(R_{11} \vee R_{12} \vee \ldots \vee R_{1 n}\right)\right\} \vee\left\{R D_{2}:\left(R_{21} \wedge R_{22} \wedge \ldots \wedge R_{2 n}\right)\right\} \vee \ldots\right.} \\
& \left.\left\{R D_{m}:\left(R_{m 1} \vee R_{m 2} \vee \ldots \vee R_{m n}\right)\right\}\right]
\end{aligned}
$$

Workflow designers specify the required role set for each task in the workflow design tool. This will be enforced by each task during the runtime. For example, if task $\mathrm{T} 1$ has a required role set as follows.

$[\{$ SchoolProject : (teacher $\vee$ instructor $)\} \vee\{$ CompanyProject : (manager $\wedge$ staff) $\}]$

A user who has the teacher, instructor, or senior roles (to teacher or instructor) in the SchoolProject role domain, or the manager and staff, or senior roles (to manager and staff) in the CompanyProject role domain is allowed to execute the task T1 and access the components or other tasks related to T1. Basically, the access control and the level of the services are based on the user's assigned roles in the enterprise's role domain. The required role set does not consider the user's organization or identity.

\subsection{DESIGN VALIDATION}

Since several portions of a workflow design may be assembled to accomplish an enterprise level mission, it is important to validate that the overall design is consistent and sound. We provide translators for converting an interorganizational workflow design into inputs to an existing Petri-net based analysis tool, Woflan [22], and a model checking tool, Spin [3], so that the consistency of the inter-organizational workflow design can be validated. Detailed mecha- 
nisms of design validation and related examples will be described in our future publications.

\section{IMPLEMENTATION SUMMARY AND CONCLUSION}

We have developed a GUI (Graphical User Interface)-based workflow design tool (described in Section 3) in JAVA. The design tool is integrated with Woflan and Spin for design validation. Currently, we are using modified OrbWork (see Section 2.1) as our runtime engine, which uses IONA's JAVA implementation of Orbix ORBs (Object Request Brokers [11]) version 3 to support CORBA in the system. To accommodate secure collaboration, OrbWork has to be extended in two major areas. The first area is to support the extended workflow interoperability model (we call it cooperative processes model) that we introduced in [8]. The second area is the incorporation of SSL that supports secure communications between clients and servers. We have integrated Phaos' SSLava [20] version 1.11 with OrbWork to provide secure communications. To support monitor functions (described in Section 3) in the system, we use MS Access via JDBC data access API [5] to store and provide transaction histories.

In this paper, we have addressed the security services for a secure workflow system to support dynamic collaboration; providing secure communications between users and system components, separating security infrastructures for organizations and their enterprises, providing different privileges to different users, and validating workflow designs. We have convinced why we need these services and described how we implemented them in our secure workflow management system. Although we have applied our ideas to particular technologies, such as workflows and RBAC, in this paper, we believe it is always possible to apply our approaches to other security systems, which support many users from different organizations.

\section{References}

[1] C. Adams. The Simple Public-Key GSS-API Mechanism (SPKM). RFC 2025, October 1996.

[2] G. Edjlali, A. Acharya, and V. Chaudhary. History-based Access Control for Mobile Code. In Proceedings of the 5th ACM Conference on Computer and Communications Security, San Francisco, CA, November 1998.

[3] G.J. Holzmann. The Model Checker Spin. IEEE Transactions on Software Engineering, Vol. 23, No.5, pp. 279-295, May 1997. See also http://cm.belllabs.com/cm/cs/what/spin/.

[4] ITU-T Recommendation X.509. Information Technology - Open Systems Interconnection - The Directory: Authentication Framework. International 
Telecommunication Union Standardization Sector, 1997.

[5] JDBC Data Access API. http://java.sun.com/products/jdbc/, Sun Microsystems, 2000.

[6] S. Kent and R. Atkinson. Security Architecture for the Internet Protocol. RFC 2401, November 1998.

[7] M. H. Kang, B. J. Eppinger, and J. N. Froscher. Tools to Support Secure Enterprise Computing. In Proceedings of 15th Annual Computer Security Applications Conference, Phoenix, Arizona, December 1999.

[8] M. H. Kang, J. N. Froscher, A. P. Sheth, K. J. Kochut, and J. A. Miller. A Multilevel Secure Workflow Management System. In Proceedings of the 11th Conference on Advanced Information Systems Engineering, Heidelberg, Germany, June 1999.

[9] M. H. Kang, J. S. Park, and J. N. Froscher. Access Control Mechanisms for Inter-organizational Workflow. In Proceedings of 6th ACM Symposium on Access Control Model and Technologies (SACMAT), Chantilly, Virginia, May 2001.

[10] K. Kochut, A. Sheth, and J. Miller. ORBWork: A CORBA-Based Fully Distributed, Scalable and Dynamic Workflow Enactment Service for METEOR. UGA-CS-TR-98-006, Technical Report, Department of Computer Science, University of Georgia, 1998.

[11] Orbix 3. http://www.iona.com/products/orbix3_home.htm, IONA, 2000.

[12] T. Parker and D. Pinkas. SESAME V4 - Overview. SESAME Technology, December 1995.

[13] J. S. Park and R. S. Sandhu. Smart Certificates: Extending X.509 for Secure Attribute Service on the Web. In Proceedings of 22nd National Information Systems Security Conference (NISSC), Crystal City, Virginia, October 1999.

[14] J. S. Park and R. S. Sandhu. Secure Cookies on the Web. IEEE Internet Computing, Vol. 4, No. 4, pp. 36-44, July-August 2000.

[15] J. S. Park and R. S. Sandhu. Binding Identities and Attributes Using Digitally Signed Certificates. In Proceedings of 16th Annual Computer Security Applications Conference (ACSAC), New Orleans, Louisiana, December 2000.

[16] J. S. Park, R. S. Sandhu, and G. J. Ahn. Role-based Access Control on the Web. ACM Transactions on Information and System Security (TISSEC), Vol. 4, No. 1, February 2001.

[17] W. Rosenberry, D. Kenney, and G. Fisher. Understanding DCE. O'Reilly \& Associates, 1992. 
[18] R. S. Sandhu, E. J, Coyne, H. Feinstein, and C. Youman. Role-based Access Control Models. IEEE Computer, Vol. 29, No. 2, pp. 38-47, February 1996.

[19] J.F. Steiner, C. Neuman, and J.I. Schiller. Kerberos: An Authentication Service for Open Network Systems. In Proceedings of Winter USENIX Conference, 1988.

[20] Phaos SSLava. http://www.phaos.com/e_security/prod-ssl.html, Phaos Technology, 2000.

[21] D. Wagner and B. Schneir. Analysis of the SSL3.0 Protocol. In Proceedings of the 2nd UNIX Workshop on Electronic Commerce, November 1996.

[22] H.M.W. Verbeek, T. Basten, and W.M.P. van der Aalst. Diagnosing Workflow Processes Using Woflan. Computing Science Report 99/02, Eindhoven University of Technology, Eindhoven, 1999. 